\title{
Interactions among eukaryotes, retrotransposons and riboviruses: endogenous riboviral elements in eukaryotic genomes
}

\author{
Masayuki Horie ${ }^{1,2 *}$ \\ ${ }^{1}$ Hakubi Center for Advanced Research, and ${ }^{2}$ Department of Virus Research, Institute for Frontier \\ Life and Medical Sciences, Kyoto University, 53 Kawahara-cho, Shogoin, \\ Sakyo-ku, Kyoto 606-8507, Japan
}

(Received 22 September 2018, accepted 7 February 2019; J-STAGE Advance published date: 29 June 2019)

\begin{abstract}
Riboviruses are viruses that have RNA genomes and replicate only via RNA intermediates. Although they do not require a DNA phase for replication and do not encode reverse transcriptase, the presence of DNA forms of riboviral sequences in ribovirus-infected cells has been reported since the 1970s. Additionally, heritable ribovirus-derived sequences, called riboviral endogenous viral elements (EVEs), have been found in the genomes of many eukaryotes. These are now thought to be formed by the reverse transcription machineries of retrotransposons within eukaryotic genomes sometimes referred to as selfish elements. Surprisingly, some reverse-transcribed riboviral DNAs (including EVEs) provide physiological functions for their hosts, suggesting the occurrence of novel interactions among eukaryotic genomes, retrotransposons and riboviruses, and opening the door to new avenues of investigation. Here I review current knowledge on these triangular interactions, and discuss future directions in this field.
\end{abstract}

Key words: endogenous viral element, evolution, retrotransposon

\section{INTRODUCTION}

Viruses are obligate parasites that infect many types of non-viral organisms and even several giant viruses (La Scola et al., 2008; Claverie and Abergel, 2009). Because virus infections can burden their hosts in several ways, non-viral organisms have gained antiviral immune systems to counteract viral infections (Daugherty and Malik, 2012; tenOever, 2016). Viruses have overcome this countermeasure by acquiring anti-immune systems (Daugherty and Malik, 2012; tenOever, 2016). These battles are thought to have been repeated numerous times, resulting in a so-called evolutionary arms race between viruses and hosts (Gammon and Mello, 2015; Hoffmann et al., 2015; tenOever, 2016). Thus, viruses are believed to have contributed to the evolution of immune systems in eukaryotes and vice versa.

Viruses are divided into four groups based on the genomic material in the virus particles (virions) and their replication modes: DNA viruses, RNA viruses (riboviruses), reverse-transcribing DNA viruses and reverse-transcribing RNA viruses. DNA viruses and

\footnotetext{
Edited by Kenji Ichiyanagi

* Corresponding author. E-mail: horie.masayuki.3m@kyoto-u.ac.jp DOI: http://doi.org/10.1266/ggs.18-00049
}

reverse-transcribing DNA viruses, and RNA viruses and reverse-transcribing RNA viruses, have DNA and RNA genomes in their respective virus particles. Although all viruses produce mRNA, the genomes of DNA viruses and RNA viruses replicate only via DNA and RNA intermediates, respectively. In contrast, reverse-transcribing DNA viruses and reverse-transcribing RNA viruses encode reverse transcriptase (RTase), which enables reverse transcription (RT). Thus, reverse-transcribing DNA and RNA viruses possess DNA and RNA as genomic material in their virions, but replicate via RNA and DNA intermediates, respectively. In this review, I focus on RNA viruses that do not encode an RTase. Because some informal definitions of "RNA viruses" include reversetranscribing RNA viruses, I have used the term "riboviruses," which explicitly excludes reverse-transcribing RNA viruses.

As described above, ribovirus particles have RNA genomes and replicate only via RNA intermediates, such as viral genomic RNA, anti-genomic RNA and mRNA. Based on the polarity of the RNA genomes in the virus particles, riboviruses are subdivided into positive-strand, negative-strand, or double-stranded RNA viruses (Flint et al., 2015). Riboviruses encode an RNAdependent RNA polymerase that makes RNA copies from RNA templates, which are necessary for their replication 
and transcription (the typical replication cycle of riboviruses is shown in Fig. 1).

Although riboviruses do not require DNA intermediates for their replication, DNA forms of riboviruses have been reported to be present in ribovirus-infected cells, beginning in 1975 (Zhdanov, 1975). Since then, several sporadic reports suggested the presence of DNA forms of riboviral sequences (Klenerman et al., 1997; Crochu et al., 2004; Maori et al., 2007), but it was unclear how the viral DNA was generated. In 2009, Geuking et al. revealed that riboviral RNA is reverse transcribed into DNA by RTase encoded by a retrotransposon (also referred to as a selfish element) present in eukaryotic genomes (Geuking et al., 2009). Additionally, we and others discovered that heritable ribovirus-derived elements exist in the genomes of eukaryotes, at least some of which were apparently reverse transcribed and integrated by retrotransposons (Belyi et al., 2010a; Horie et al., 2010; Katzourakis and Gifford, 2010). These ribovirus-derived sequences are believed to have originated from reverse-transcribed and integrated riboviral sequences in the hosts' germline cells. Such heritable virus-derived sequences are now known as endogenous viral elements (EVEs) (Katzourakis and Gifford, 2010). After these findings were reported, many other studies showed the presence of riboviral EVEs derived from diverse viruses in eukaryotic genomes (Horie and Tomonaga, 2011; Aiewsakun and Katzourakis, 2015; Parrish and Tomonaga, 2016).

Surprisingly, ribovirus-derived DNA is not just a byproduct of chance interactions between riboviruses and retrotransposons: at least in insects, these interactions play important roles in antiviral immunity (Goic et al., 2013, 2016; Tassetto et al., 2017; Poirier et al., 2018). Additionally, some ribovirus-derived EVEs also play roles in their hosts (Fujino et al., 2014; Myers et al., 2016). These findings provide support for a novel concept regarding biological interactions among riboviruses, retrotransposons and eukaryotic genomes. In this review, I focus on such ribovirus-retrotransposon interactions in animals, and summarize and discuss the modes of their interactions and biological functions in host animals.

\section{INTERACTIONS BETWEEN RIBOVIRUSES AND RETROTRANSPOSONS}

As mentioned above, riboviral RNA can be reverse transcribed into DNA in some ribovirus-infected cells (Zhdanov, 1975; Klenerman et al., 1997; Geuking et al., 2009; Horie et al., 2010; Goic et al., 2013, 2016; Tassetto et al., 2017; Poirier et al., 2018). Because riboviruses only encode an RNA-dependent RNA polymerase, an RTase must be provided in trans for the RT reactions of riboviruses. Several elements, such as retroelements, and telomerase are known to have RTase activity in eukaryotic cells. Additionally, coinfection with retroviruses, which are reversetranscribing RNA viruses encoding RTase and capable of integration, can also serve as sources of RTase. Among them, retroelements and possible co-infection with a retrovirus are reported to be involved in the RT reactions and, in some cases, the integration of riboviral sequences.

Retrotransposons are transposable elements that can expand in a copy-and-paste manner within genomes: retrotransposons are transcribed as RNA, which is reverse transcribed into cDNA, and then integrated into the genome (Fig. 2A). Based on the presence or absence of long terminal repeats (LTRs), retrotransposons are classified as LTR and non-LTR retrotransposons, both of which are further divided into autonomous and nonautonomous classes (Havecker et al., 2004; Eickbush and Jamburuthugoda, 2008). While autonomous retrotransposons encode RTase, which enables retrotransposition in cis, non-autonomous retrotransposons do not

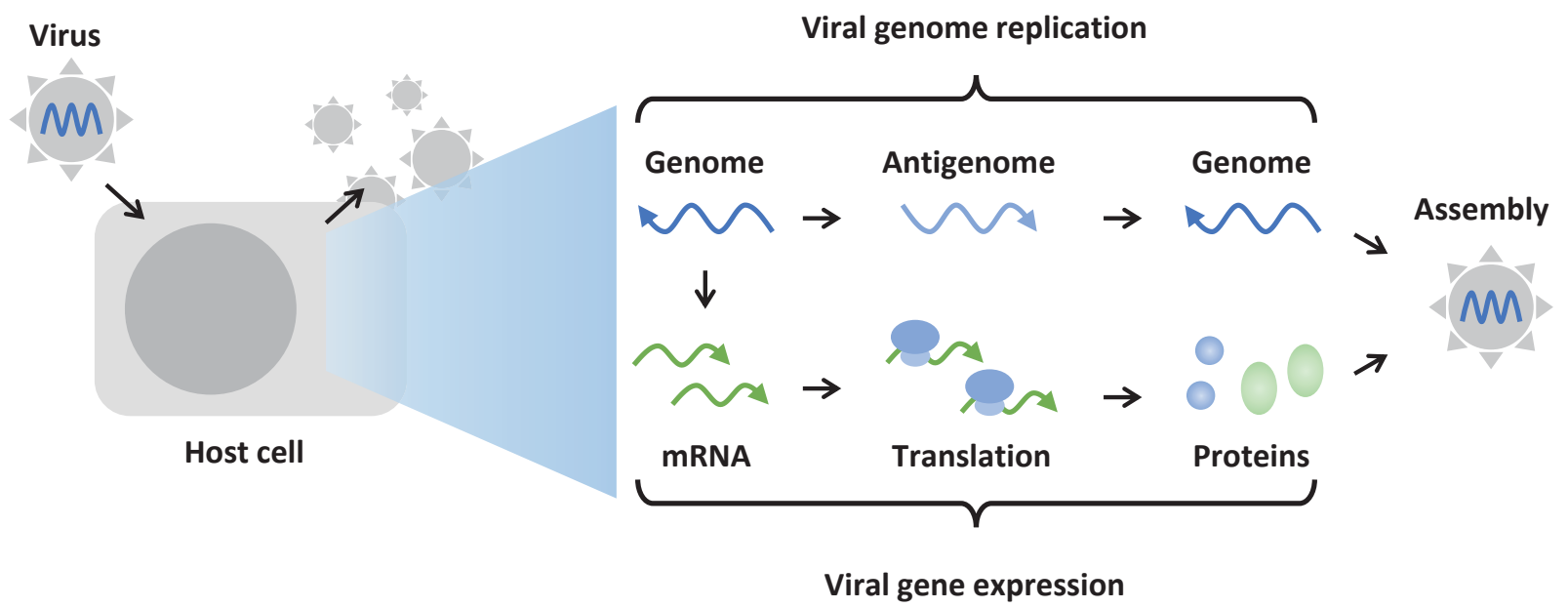

Fig. 1. Replication cycle of a ribovirus with negative polarity. A schematic representation of the replication of a ribovirus with a negative-strand RNA genome. 
A

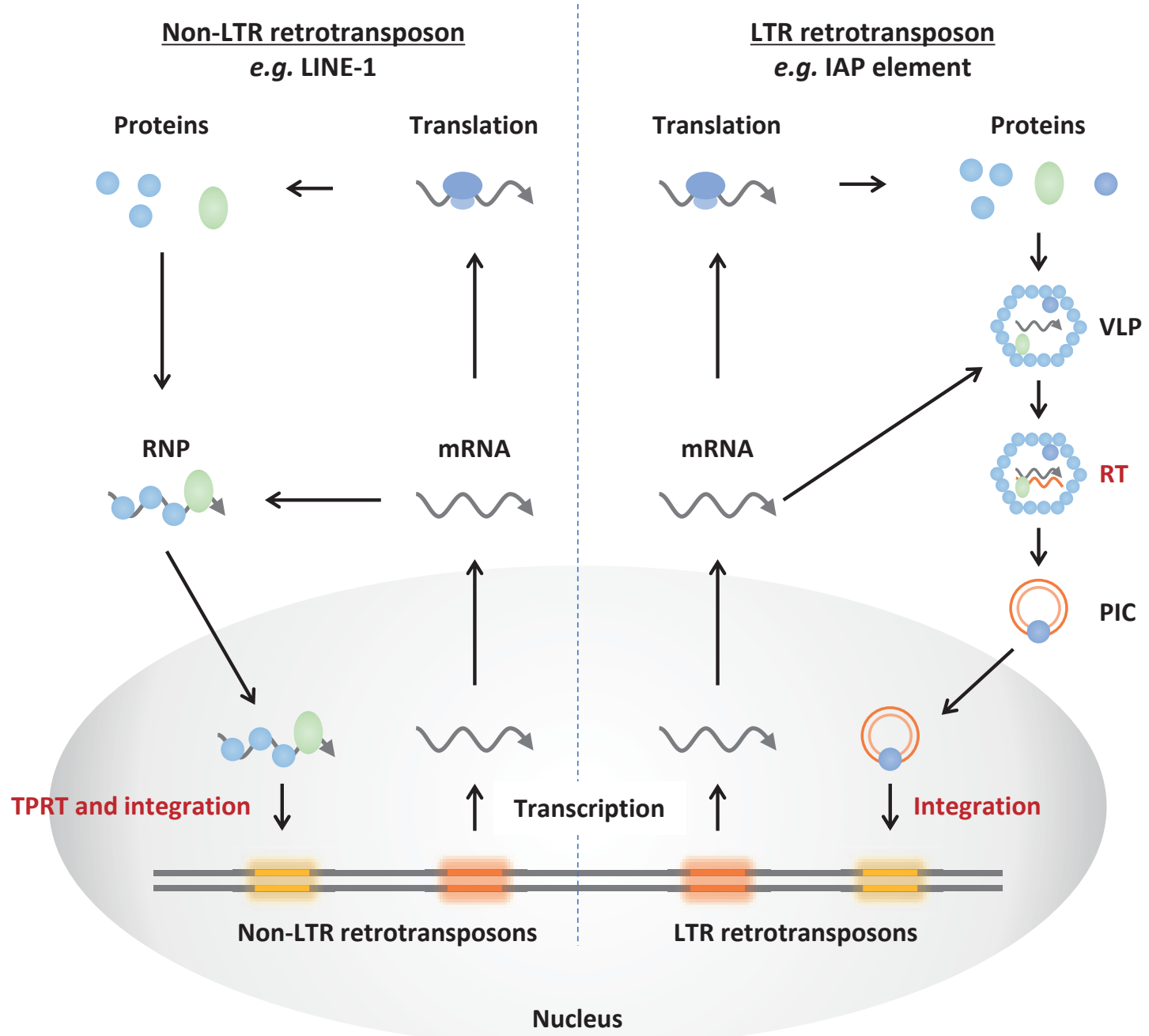

B

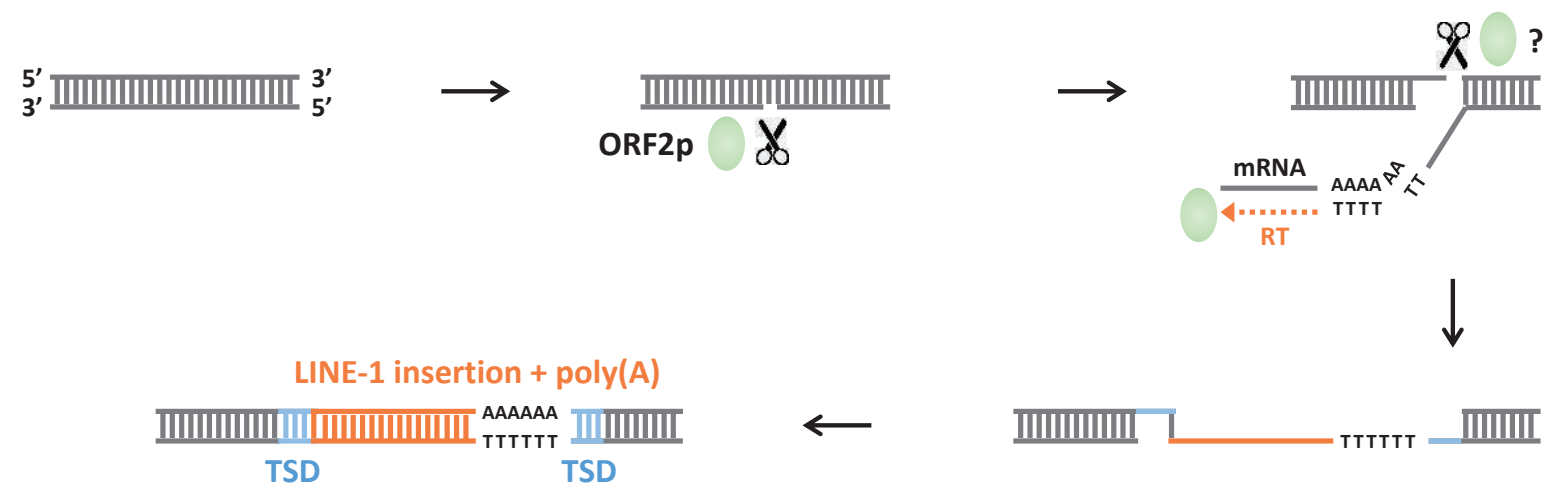

Fig. 2. Modes of retrotransposition of non-LTR and LTR retrotransposons. (A) Schematic representations of retrotranspositions are shown. PIC, pre-integration complex; RNP, ribonucleoprotein; RT, reverse transcription; VLP, virus-like particle; TPRT, target-primed reverse transcription. (B) A schematic representation of a LINE-1 retrotransposition is shown. RT, reverse transcription; TSD, target site duplication. 
encode RTase, and thus must exploit the machineries of autonomous retrotransposons for their retrotransposition (Beauregard et al., 2008).

LTR retrotransposons have, as the name suggests, LTRs at both ends, which usually contain several elements essential for retrotransposition, such as a promoter and primer-binding sites (Havecker et al., 2004). LTR retrotransposons harbor at least two genes, namely a gag and pol gene. The gag gene encodes structural proteins that form virus-like particles together with retrotransposon RNAs. The pol gene encodes a protease, integrase and RTase, which catalyze retrotransposition (Havecker et al., 2004). Some LTR retrotransposons, including endogenous retroviruses, also have an env gene that enables the formation of infectious particles and subsequent transmission to other cells (Malik et al., 2000; Havecker et al., 2004). As mentioned above, LTR retrotransposons form virus-like particles in cells where retrotransposon RNAs are reverse-transcribed into DNA using tRNA as primers (Havecker et al., 2004). Next, a retrotransposon DNAintegrase complex (also called the pre-integration complex) is formed, which is translocated into the nucleus and is integrated into a chromosome via integrase activity (Fig. 2A) (Havecker et al., 2004). Therefore, as suggested by their RT, LTR retrotransposons are also known as extrachromosomally primed retrotransposons (Beauregard et al., 2008). LTR retrotransposons comprise a large part of animal genomes; for example, they account for $8 \%$ of the human genome (IHGSC, 2001). Some LTR retrotransposons are non-autonomous. These non-autonomous LTR retrotransposons lack essential genes for retrotransposition, and thus they exploit the machineries of autonomous retrotransposons (Havecker et al., 2004).

Non-LTR retrotransposons usually do not have LTRs (Beauregard et al., 2008). As described above, non-LTR retrotransposons are also further divided into autonomous and non-autonomous classes. Autonomous nonLTR retrotransposons encode two proteins: ORF1p has RNA chaperone activity, and ORF2p possesses RTase and endonuclease activities (Feng et al., 1996; Martin, 2006; Beauregard et al., 2008). ORF1p and ORF2p interact with retrotransposon RNA to form a ribonucleoprotein (RNP) complex (Beauregard et al., 2008). The RNP complex is translocated into the nucleus, after which the encoded endonuclease nicks the chromosomal DNA and initiates RT using the nicked DNA as a primer (Beauregard et al., 2008). This mode of RT is known as target-primed reverse transcription (TPRT), and thus non-LTR retrotransposons are also referred to as targetprimed retrotransposons (Beauregard et al., 2008). In addition to ORF1p and ORF2p, long interspersed nuclear element 1 (LINE-1) in primates, an autonomous non-LTR retrotransposon that is still active in humans, encodes ORF0p in the antisense orientation, which affects the mobility of LINE-1 (Denli et al., 2015). In contrast, non- autonomous non-LTR retrotransposons are retrotransposed via the machineries of autonomous non-LTR retrotransposons. Non-LTR retrotransposons represent a major component of eukaryotic genomes; for example, approximately $30 \%$ of the human genome is comprised of non-LTR retrotransposons (IHGSC, 2001).

Retrotransposons are still active in the genomes of many eukaryotes, including humans (Mills et al., 2007; Hancks and Kazazian, 2016). Indeed, several genetic disorders are caused by de novo insertions of retrotransposons (Hancks and Kazazian, 2012, 2016). Thus, retrotransposons are also referred to as selfish genetic elements. However, they are also considered as driving forces of genome evolution, because retrotransposition can cause genome rearrangement, provide gene regulators, and generate chimeric genes (Cordaux and Batzer, 2009). Some retrotransposon genes may be co-opted by the host cell. For example, the Peg10, Peg11 and Syncytin genes, which are derived from LTR retrotransposons (endogenous retroviruses), are essential for placental formation in mammals (Mi et al., 2000; Dupressoir et al., 2005; Ono et al., 2006; Sekita et al., 2008). Additionally, non-LTR retrotransposons have been implicated in the formation of processed pseudogenes and retrocopies of cellular mRNAs (Esnault et al., 2000). Namely, cellular mRNAs are occasionally incorporated into nonLTR retrotransposon machineries, and then reverse transcribed and integrated into a different position within the genome. Thus, retrotransposons have contributed to the evolution of genomes in various ways.

Although RT and integration of riboviral sequences were first reported in the 1970s (Zhdanov, 1975), the underlying mechanisms have remained mysterious because riboviruses themselves do not encode RTase. However, a series of studies revealed that retrotransposons are involved in RT of riboviral RNAs; furthermore, in some cases, the retrotransposons play a role in capturing foreign viral genetic elements (Geuking et al., 2009; Belyi et al., 2010a; Horie et al., 2010, 2013; Horie and Tomonaga, 2011; Goic et al., 2013; Poirier et al., 2018). In this section, I focus on the RT/integration of riboviral sequences by retrotransposable elements.

RT of riboviral sequences by non-LTR retrotransposons in somatic cells Some riboviral RNAs have been suggested to be reverse transcribed and integrated into host genomes by the RTase activities of the non-LTR retrotransposon LINE-1 in somatic cells (Horie et al., 2010, 2013; Shimizu et al., 2014).

LINE-1 retrotransposons are autonomous non-LTR retrotransposons, and some of them are active in many species of animals, including humans (Sassaman et al., 1997; Brouha et al., 2003). As described in the above section, RT and LINE-1 insertion are initiated by cleavage of single-stranded DNA by LINE-1 ORF2p (Fig. 2B). The $3^{\prime}$ 
end of the cleaved DNA is used as a primer for RT. The opposite DNA strand is also cleaved, but the cleavage site is usually located downstream of the first DNA strand, which eventually generates a short direct repeat, referred to as a target site duplication (TSD), on both sides of the inserted LINE-1 (the mode of LINE-1 retrotransposition is reviewed by Beck et al., 2011). Importantly, LINE-1 is involved in the formation of processed pseudogenes, and the resultant integrants have features similar to those observed in retrotransposed LINE-1 (Esnault et al., 2000).

We previously reported that the mRNA of Borna disease virus (BoDV), a ribovirus, was reverse transcribed into DNA, at least a portion of which was integrated into the host chromosome by the machinery of LINE-1 (Horie et al., 2010). BoDV is a negative-strand RNA virus, belonging to the family Bornaviridae, which establishes non-cytolytic persistent infections in the host cell nucleus (Tomonaga et al., 2002). Previously, we detected virusderived DNA (vDNA) in BoDV-infected cultured cells from diverse mammalian species (such as humans, rabbits, dogs and mink), as well as in mouse brains in vivo (Horie et al., 2010). We also demonstrated, using AluPCR and inverse PCR, that BoDV vDNA was integrated into host chromosomes (Horie et al., 2010, 2013). We identified the integrated BoDV DNAs and their flanking sequences, revealing that many of the integrants have structures similar to cellular processed pseudogenes: the integrants had poly $(\mathrm{A})$ tails, short tandem repeats derived from host sequences (e.g., TSDs observed upstream and downstream of the integrants), and occasional $5^{\prime}$ truncations of mRNA (Gilbert et al., 2002). These observations strongly suggested that BoDV vDNA was derived from mRNA following RT by the LINE-1 machinery. In addition to such typical sequences, we also observed integrants that lacked a poly(A) tail, and displayed deletions of flanking sequences and reorganization of flanking sequences (Horie et al., 2010, 2013). These differences may reflect non-canonical integration or endonucleaseindependent retrotransposition, as observed for LINE-1 retrotransposition (Gilbert et al., 2002; Morrish et al., 2002). We further showed that the integration efficiency of BoDV mRNA was very low in two human cell lines: less than $0.1 \%$ (below the detection limit) of BoDV-infected cells have integrants originating from BoDV mRNA (Horie et al., 2010, 2013). However, it is unclear whether BoDV mRNA is preferentially recognized by the LINE-1 machinery.

Vesicular stomatitis virus (VSV), a ribovirus belonging to the family Rhabdoviridae (Amarasinghe et al., 2018), is also reverse transcribed by the LINE-1 machinery (Shimizu et al., 2014). VSV replicates in the cytoplasm and causes cytopathic effects in infected cells (Shimizu et al., 2014). Shimizu et al. (2014) detected VSV-derived DNA in VSV-infected human cells. The VSV vDNA was suggested to be present as single-stranded DNA molecules of antisense orientation in the cytoplasm, as determined by fractionation and PCR. The authors attempted to determine the sequence of VSV vDNA by inverse PCR and sequencing analyses, using essentially the same method as for our BoDV study. However, they could only detect virus-derived sequences, and no host-derived sequences: all the identified sequences corresponded to a full-length viral mRNA sequence including a poly(A) tail (Shimizu et al., 2014). Based on these observations, they proposed that in the cytoplasm, VSV mRNA alone is reverse transcribed and that the vDNA is present as extrachromosomal DNA (Shimizu et al., 2014). However, the source of primers for RT is unknown in this model. Shimizu et al. (2014) suggested that special DNA primers are not needed for the RT, or that oligo $\mathrm{U}$ or $\mathrm{T}$ are used as the primers. An alternative explanation is that the detected vDNAs were derived from first-strand cDNA fragments whose second-strand DNA was not yet synthesized. Several cellular factors are involved in LINE-1-mediated TPRT and integration (Pizarro and Cristofari, 2016). Thus, although firststrand VSV cDNA is synthesized in VSV-infected cells, the viral infection induces apoptosis, which may disrupt the cellular machineries for synthesizing second-strand DNA; the cDNA may then be digested by a cellular DNase and leak into the cytoplasm. Indeed, VSV infection causes fragmentation of the host chromosomal DNA (Koyama, 1995). Furthermore, the relative amounts of VSV vDNA and leaked nuclear genomic DNA in the cytoplasm were very similar (Shimizu et al., 2014). Further studies are needed to understand the mode of RT of VSV. For example, several host factors were involved in apoptosis induced by VSV infection (Pearce and Lyles, 2009). Importantly, knockdown of some of these factors inhibited VSV-induced apoptosis. Thus, such systems may be useful to pursue this issue.

Shimizu et al. (2014) also detected vDNA derived from respiratory syncytial virus and echovirus type 35, which are riboviruses with negative- and positive-strand RNA genomes, respectively. These observations suggest that many riboviruses are potentially reverse transcribed by the LINE-1 machinery. However, the biological significance of the RT of riboviruses is unclear. Additionally, it is unclear whether the RT of these riboviruses occurs in vivo. In somatic cells, retrotransposons are usually inactive except in several types of somas, such as neuronal progenitor cells (Muotri et al., 2005; Yang and Kazazian, 2006; Coufal et al., 2009; Garcia-Perez et al., 2010). Therefore, riboviruses that do not infect such cells may not be reverse transcribed by the LINE-1 machinery. In this regard, in vivo animal experiments, as we performed for BoDV in mice (Horie et al., 2010), are also important for obtaining a deeper understanding of the authentic interactions between riboviruses and retrotransposons. 
Germline-integrated riboviral sequences in eukaryotic genomes, presumably reverse transcribed and integrated by non-LTR retrotransposons In addition to the RT of extant viruses, we and others revealed that sequences homologous to riboviruses, such as bornaviruses and filoviruses, are present in eukaryotic genomes (Belyi et al., 2010b; Horie et al., 2010). These sequences are believed to be derived from reverse-transcribed and germline-integrated sequences of riboviruses, known as riboviral EVEs. The RT and integration steps are likely mediated by the LINE-1 machinery because some of the bornaviral and filoviral EVEs have poly(A) tails and TSDs, as observed for the integrants of a modern bornavirus, BoDV (Belyi et al., 2010b; Horie et al., 2010). Thus, retrotransposons may have contributed to capturing foreign genetic material from ancient riboviruses.

Structures of integrants should provide insights into the modes of RT and the characteristics of ancient viruses (Horie and Tomonaga, 2019). Among the abovementioned riboviral EVEs, many are thought to have been integrated by TPRT of viral mRNAs alone. However, intriguingly, an endogenous bornavirus-like element (EBL) in humans (named hsEBLN-2) seems to have been reverse transcribed and integrated as a recombinant between viral mRNA and a non-autonomous retrotransposon, the Alu element (Belyi et al., 2010b; Horie et al., 2010). An Alu element was identified immediately downstream of the poly(A) tail of hsEBLN-2. Importantly, the hsEBLN-2/Alu element is flanked by a 9-bp TSD, and the Alu element itself is not flanked by a TSD (Fig. 3). This may have been caused by a template switch from the Alu RNA to mRNA of an ancient bornavirus during TPRT of the Alu. This observation suggests that there are two modes of LINE-1-mediated integration of riboviral sequences, as follows. First, viral mRNA is directly reverse transcribed and integrated into a chromosome by the LINE-1 machinery. In this case, viral mRNA may be accidentally incorporated into RNP in the cytoplasm, and then translocated into the nucleus followed by TPRT of viral mRNA. Second, a template switch from a retrotransposon RNA to a viral mRNA occurs during a TPRT reaction of the retrotransposon, leading to integration of a chimeric retrotransposon-viral mRNA sequence (Fig. 3). This scenario is interesting in that it may yield novel insights into the replication sites of ancient viruses. LINE-1-mediated TPRT initiates in the cell nucleus; thus, in theory, only viruses being transcribed in the nucleus have the potential to be integrated by the template-switching mechanism. Indeed, a substantial portion of reported chimeric retrocopies, which were suggested to be formed by template switches between retrotransposon RNAs and other species of RNAs, are composed of the following elements: 5 ' parts derived from RNA localizing in the nucleus or in both nucleus and cytoplasm, and 3 ' parts originating from cytoplasmic mRNAs of cellular genes or of retrotransposons (Buzdin et al., 2002; Buzdin et al., 2003; Buzdin, 2004). Generally, few lineages of riboviruses (such as bornaviruses) are known to replicate and undergo transcription in the nucleus (Horie and Tomonaga, 2019). Currently, we have only a limited understanding regarding the replication sites of ancient riboviruses: only one lineage of bornavirus was suggested to have replicated in the cell nucleus (Horie and Tomonaga, 2019). However, hsEBLN-2 seems to have been endogenized by the template-switching mechanism described above, suggesting that an ancient bornavirus replicated in the host cell nucleus. By comparing orthologs of hsEBLN-2 in platyrrhines, their integration time was estimated to be more than 40 million years ago (MYA) (Horie et al., 2010). Therefore, this lineage may also have replicated and been transcribed in the nucleus more than 40 MYA. Although many ribovirus-derived EVEs have been reported, only a few studies focused on biological characteristics of ancient riboviruses (Horie and Tomonaga, 2019). Thus, further detailed sequence analyses of such ancient riboviruses should contribute to a better understanding of the replication mechanisms of ancient viruses. An increase in the available genome data would also be helpful to pursue this open question. Furthermore, a combination of reconstruction of ancient viral sequences, as reported for dependoparvovirus-like elements (Smith et al., 2016), and biochemical and molecular biological experiments would be useful to understand the biological features of ancient riboviruses.

RT of riboviral sequences by LTR retrotransposons in somatic cells Many riboviral sequences have also been shown to be reverse transcribed and (in some cases) integrated into the host genome by LTR retrotransposons. Lymphocytic choriomeningitis virus (LCMV) is an ambisense RNA virus belonging to the genus Mammarenavirus of the family Arenaviridae. In 1997, Klenerman et al. reported the presence of DNA forms of LCMV in LCMV-infected cells (Klenerman et al., 1997). Later, Geuking et al. (2009) reported that LCMV RNA was reverse transcribed and integrated into host chromosomes by a murine LTR retrotransposon, namely an intracisternal A-particle (IAP) retrotransposon.

Over 1,000 IAP retrotransposons are present in the mouse genome, some of which are actively retrotransposed (Kuff and Lueders, 1988). The IAP encodes three genes (gag, prt and pol genes), all of which are required for retrotransposition (Dewannieux et al., 2004). The gag gene encodes structural proteins, which form viruslike particles within cells. The prt gene encodes a protease that is involved in proteolytic cleavage of the IAP gene products. The pol gene encodes RTase and integrase. The mode of IAP retrotransposition is summarized in the section " 2 . Interactions between riboviruses and retrotransposons" and in Fig. 2A. 


\section{A}
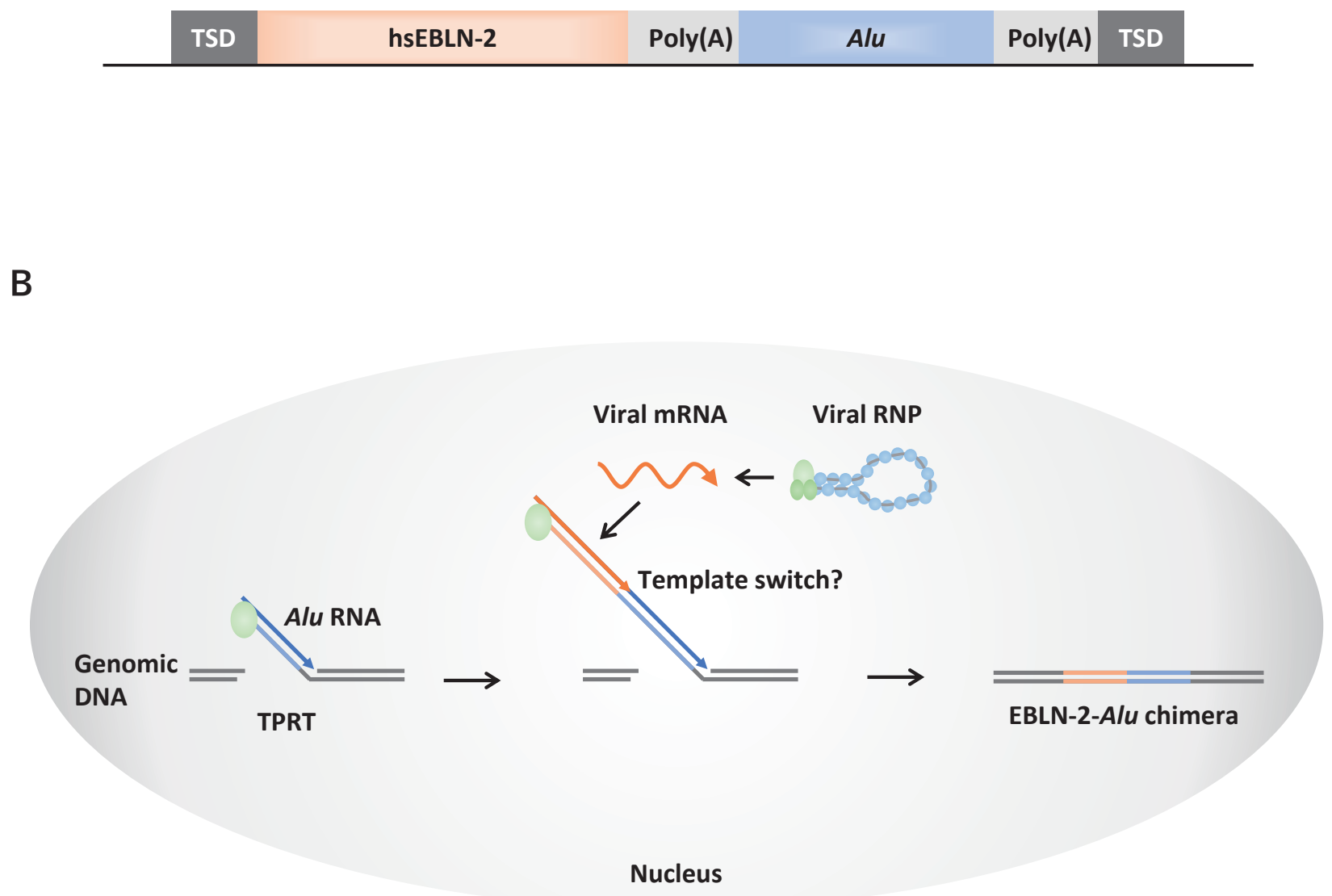

Fig. 3. Possible reverse transcription and integration mechanism of an endogenous bornavirus-like element. (A) Schematic representation of the organization of a target site duplication (TSD). hsEBLN-2, Homo sapiens endogenous bornavirus-like element 2; Poly(A): polyadenine stretch. (B) Schematic representation of possible reverse transcription and integration mechanisms of the chimeric hsEBLN-2-Alu-Sx sequence. TPRT, target-primed reverse transcription.

LCMV DNA was detected as recombinants of LCMV and IAP, and at least a portion of the LCMV/IAP chimeric DNA was present as a chromosomally integrated sequence. This recombination is, at least in IAPexpressing HeLa cells (a human cell line), likely to be mediated by a copy-choice mechanism (Geuking et al., 2009). Interestingly, integration of the LCMV/IAP DNA seemed to be achieved through an unusual process, because the integrants lacked TSDs in flanking regions, which are usually observed when integration into the genome occurs through the canonical integration mechanisms of LTR retrotransposons, and deletion of integration sites was observed (Geuking et al., 2009). Based on these observations, the authors proposed that integration of LCMV/IAP recombinants had occurred at sites of DNA damage (Geuking et al., 2009). Thus, some portion of LCMV/IAP DNA that is present as extrachromosomal vDNA may be coincidentally integrated into chromosomes at sites of DNA damage. Interestingly, LCMV vDNA formation could also be mediated by another RTase: human immunodeficiency virus RTase also supported the formation of LCMV vDNA, although less efficiently than IAP (Geuking et al., 2009). In contrast, no LCMV DNA was detected in the guinea pig cell line CCL158, which has very high RTase activity (Geuking et al., 2009). Thus, some riboviruses and RTase complexes may be compatible (Horie and Tomonaga, 2011). Sequence specificities of RTase and riboviral sequences and/or spaces for the replication of riboviruses and the formation of LTR retrotransposon virus-like particles are probably involved in such compatibility.

Many other riboviruses are also reverse transcribed into vDNA in insect cells. For example, several reports showed that riboviruses with positive- or doublestranded RNA, such as Flock House virus (FHV), Drosophila $\mathrm{C}$ virus, Drosophila $\mathrm{X}$ virus, and Sindbis virus, 
are reverse transcribed into DNA in flies and mosquitoes (Goic et al., 2013, 2016; Tassetto et al., 2017; Poirier et al., 2018). Goic et al. (2013) determined the nucleotide sequences of FHV vDNAs in FHV-infected Drosophila cells, showing that vDNAs were present as chimeric sequences with diverse LTR retrotransposons. This finding suggests that the RT reactions in flies were also catalyzed by the RTase activity of LTR retrotransposons (Goic et al., 2013). Importantly, Tassetto et al. (2017) showed that vDNA is produced in hemocytes in Drosophila. Furthermore, Poirier et al. (2018) revealed that defective viral genomes, which are derived from discontinuous viral genomic sequences, are preferentially reverse transcribed into vDNA by LTR retrotransposons. Interestingly, some of the reverse-transcribed vDNA was present as circular forms (circular vDNA: cvDNA). Other than RTases encoded by retrotransposons, two cellular factors (Ago2 and Dicer-2) were shown to be involved in vDNA formation in fruit flies (Tassetto et al., 2017; Poirier et al., 2018). The roles of both factors in RNA interference (RNAi) are well documented: Ago2 is the core effector of the RNA-induced silencing complex, and the RNase activity of Dicer-2 cleaves dsRNA into small interfering RNA (siRNA) (Wilson and Doudna, 2013). Tassetto et al. (2017) showed that Ago2 is involved in the formation of vDNA. As described above, vDNA was shown to be generated in hemocytes of Drosophila. This was demonstrated by generating a mutant lacking Ago2 in hemocytes, leading to no vDNA production during a virus infection. Poirier et al. (2018) showed that Dicer-2 was important for the formation of vDNA. They also identified the helicase domain of Dicer-2 as being important for the generation of vDNA. The helicase domain of Dicer-2 interacted with viral RNA, and mutations in Dicer-2 modified the amount of vDNA in infected cells. However, for both factors, the detailed mechanisms remain unclear.

For mosquito cells, detailed sequencing analysis has not yet been performed. Interestingly, however, at least a portion of vDNA also existed as extrachromosomal cvDNA in mosquito cells, and seemed to be transcribed from defective viral genomes (Poirier et al., 2018). In all of the chimeric virus-retrotransposon cvDNA sequences identified, the virus-derived sequences consisted of non-continuous viral sequences. Additionally, deep sequencing analysis showed that approximately half of the cvDNA-derived reads were non-continuous viral sequences. However, the detailed mechanisms underlying the formation of cvDNA are also unclear in mosquitoes.

Germline-integrated riboviral sequences in eukaryotic genomes, presumably reverse transcribed and integrated by LTR retrotransposons Some riboviral EVEs seem to have been endogenized by LTR retrotransposons (Ballinger et al., 2012; Cotton et al., 2016; Whitfield et al., 2017). This is especially obvious in insects. Ballinger et al. (2012) found that EVEs derived from sigmavirus, a ribovirus belonging to the family Rhabdoviridae, are present in the Drosophila genome, and appear to have been integrated by a blastopia-like LTR retrotransposon. Several copies of sigmaviral EVEs in Drosophila were detected as sigmavirus-LTR retrotransposon hybrid sequences. The sigmaviral EVEs have poly(A) tails, indicating that they were derived from mRNA. Although the detailed mechanism is unclear, these observations suggest that viral mRNA was reverse transcribed by a template-switching mechanism of an LTR retrotransposon. Interestingly, two sigmaviral EVEs were found to be embedded in LTR retrotransposons, suggesting that template switching occurred twice. Such an event seems to be rare, but Ballinger et al. (2012) gave a plausible explanation: elements with two LTRs may tend to be integrated into chromosomes.

Whitfield et al. (2017) also analyzed riboviral EVEs in the genome of Aedes mosquito-derived cultured cells. They utilized long-read sequencing technology to determine the genomic sequence of Aga2 cells, which are cultured cells derived from Ae. aegypti (Whitfield et al., 2017). Using the resultant high-quality contigs, they identified EVEs derived from eight families of riboviruses having positive- and negative-strand RNA genomes. Among them, flavivirus-, rhabdovirus- and chuvirus-derived EVEs accounted for a large proportion of the detected EVEs. They also analyzed elements flanking the EVEs, revealing that many of the flaviviral and rhabdoviral EVEs are flanked by LTR retrotransposons, especially by Ty3/Gypsy retrotransposons. The deep relationship between viruses and Ty3/Gypsy retrotransposons raises the possibility that compatibility exists between viruses and retrotransposons, as observed for LCMV and the IAP element (Geuking et al., 2009) (see section 2-3). However, this might also be a product of chance: Ty3/Gypsy elements may simply have been very active when the EVEs were integrated (Whitfield et al., 2017). Chuviral EVEs are flanked by a Pao Bel element (a type of LTR retrotransposon). Intriguingly, chuviral EVEs are mainly surrounded by one specific lineage of Pao Bel element, known as Pao Bel element 179 (Whitfield et al., 2017). This finding suggests that chuviral EVEs exist as a part of Pao Bel element 179 (Whitfield et al., 2017). Indeed, some LTR retrotransposons were proposed to have acquired envelope-like genes from viral glycoprotein genes (Malik et al., 2000). Unfortunately, Whitfield et al. did not perform detailed analyses for the Pao Bel element 179 or the chuviral EVEs. Further research will lead to a better understanding of the relationship between retrotransposons and riboviruses in insects.

A nodavirus-like EVE was also suggested to have been endogenized into the nematode Bursaphelenchus xylophilus genome by an LTR retrotransposon (Cotton et 
al., 2016). Nodaviruses belong to the family Nodaviridae and are positive-strand RNA viruses that replicate in the cytoplasm. Cotton et al. (2016) found a nodaviral RNA-dependent RNA polymerase-like element, named eBxnv-1, in the genome of B. xylophilus. Similar to the above example of sigmaviral EVEs (Ballinger et al., 2012), eBxnv-1 was also detected as a nodavirus-LTR retrotransposon hybrid sequence: eBxnv-1 was embedded in an LTR retrotransposon (Cotton et al., 2016). Thus, this EVE was also suggested to be integrated via recombination between viral RNA and an LTR retrotransposon.

In addition to the above findings, a bornavirus-derived EVE (named itEBLN) in the thirteen-lined ground squirrel may have been endogenized by an LTR retrotransposon. We previously estimated that itEBLN was integrated within 0.3 MYA (Suzuki et al., 2014). Importantly, LINE-1 activity became extinct approximately 4-5 MYA in this lineage (Platt and Ray, 2012). Thus, the endogenization of itEBLN probably occurred after the extinction of LINE-1, suggesting that RTase was provided by a retrotransposon other than LINE-1; the candidate element is an LTR retrotransposon. Although no obvious recombination was found, two LTR retrotransposons and a SINE (short interspersed nuclear element; a non-autonomous non-LTR retrotransposon) are located immediately upstream of itEBLN (Suzuki et al., 2014). Because retrotransposition of an LTR retrotransposon occurred 0.3 MYA in this lineage, itEBLN might have been integrated as recombinants of the LTR retrotransposons, the SINE and a bornaviral transcript (Suzuki et al., 2014). Further studies are needed to understand how itEBLN was endogenized. Since itEBLN was estimated to be integrated very recently, sequencing analyses of syntenic loci in closely related species should provide clues about the endogenization mechanism of itEBLN.

\section{BIOLOGICAL FUNCTIONS OF RIBOVIRAL RNA RT IN SOMATIC CELLS}

As described above in section 2, ribovirus-derived RNA can be reverse transcribed into vDNA in somatic cells. Such reverse-transcribed riboviral DNA may be a byproduct resulting from coincidental interactions between riboviruses and retrotransposons. However, at least in several insects, such as fruit flies and mosquitoes, $\mathrm{RT}$ of riboviral RNA during infection seems to be very important for antiviral defenses (Fig. 4).

In Drosophila, reverse-transcribed vDNA is important for small RNA-mediated antiviral immunity (Goic et al., 2013; Tassetto et al., 2017; Poirier et al., 2018). Goic et al. (2013) showed that reverse-transcribed DNA is important for resistance against exogenous virus infections. As described above in sections 2 and 3, FHVderived vDNA was detected in FHV-infected fruit flies as highly reconstructed DNA: the sequences originated from non-continuous viral sequences (Goic et al., 2013). Further analyses showed that vDNAs are present as chimeras with LTR retrotransposons. Small RNA-seq analyses revealed that many siRNAs could be mapped to the chimeric sequences, suggesting that siRNAs are expressed from the vDNA-retrotransposon chimeras. Treatment with the RTase inhibitor azidothymidine (AZT) decreased vDNA formation and increased viral titers as well as mortality of infected flies. Thus, Goic et al. (2013) concluded that vDNA formation is important for resistance against exogenous virus infection in Drosophila, and proposed that vDNAs can serve as templates for antiviral siRNA production.

Tassetto et al. (2017) also showed the importance of vDNA formation for systemic antiviral defense in Drosophila. They found that viral RNA in hemocytes is reverse transcribed into vDNA, which can act as a template for antiviral siRNA synthesis. The produced siRNA was released into extracellular exosome-like vesicles to promote systemic antiviral RNAi in infected individuals. Interestingly, the vDNA was present for more than 21 days, which corresponds to one-third of the lifespan of a fly. Thus, Tassetto et al. (2017) proposed that vDNA formation is involved in immune memory for systemic immunity in Drosophila. Intriguingly, hemocytes themselves did not seem to be efficiently infected by the viruses, and thus virus-derived dsRNA was proposed to be provided from other infected cells. Indeed, a dsRNAuptake system that may contribute to this type of immunity has been reported (Saleh et al., 2006, 2009).

Poirier et al. (2018) further showed that in Drosophila, defective riboviral genomes tend to be reverse transcribed and the resultant cvDNA can serve as a template for antiviral siRNA production. Furthermore, they found that Dicer-2 played a dual role in this antiviral system: the well-known function of Dicer-2 is to cleave dsRNA into siRNA, but it also functions as a pattern recognition receptor (PRR) that senses foreign nucleic acids. As described above, the helicase domain of Dicer-2 is involved in the formation of cvDNA and interacts with viral RNA. Thus, the authors proposed that Dicer-2 acts as a PRR analogous to RIG-I-like receptors in mammals, which bind viral dsRNAs and activate innate immune signaling pathways. Taken together, these findings suggest that in Drosophila, vDNA formation is involved in small RNA-mediated anti-riboviral immunity (Poirier et al., 2018).

In Aedes mosquitoes, vDNA formation is involved in tolerance to virus infections. This is different from Drosophila, in which vDNA formation was shown to be important for resistance against virus infection. Goic et al. (2016) showed that vDNA was generated in Chikungunya virus- or Dengue virus-infected mosquito cells. Treatment with the RTase inhibitor AZT blocked vDNA formation, leading to reduced small RNA produc- 


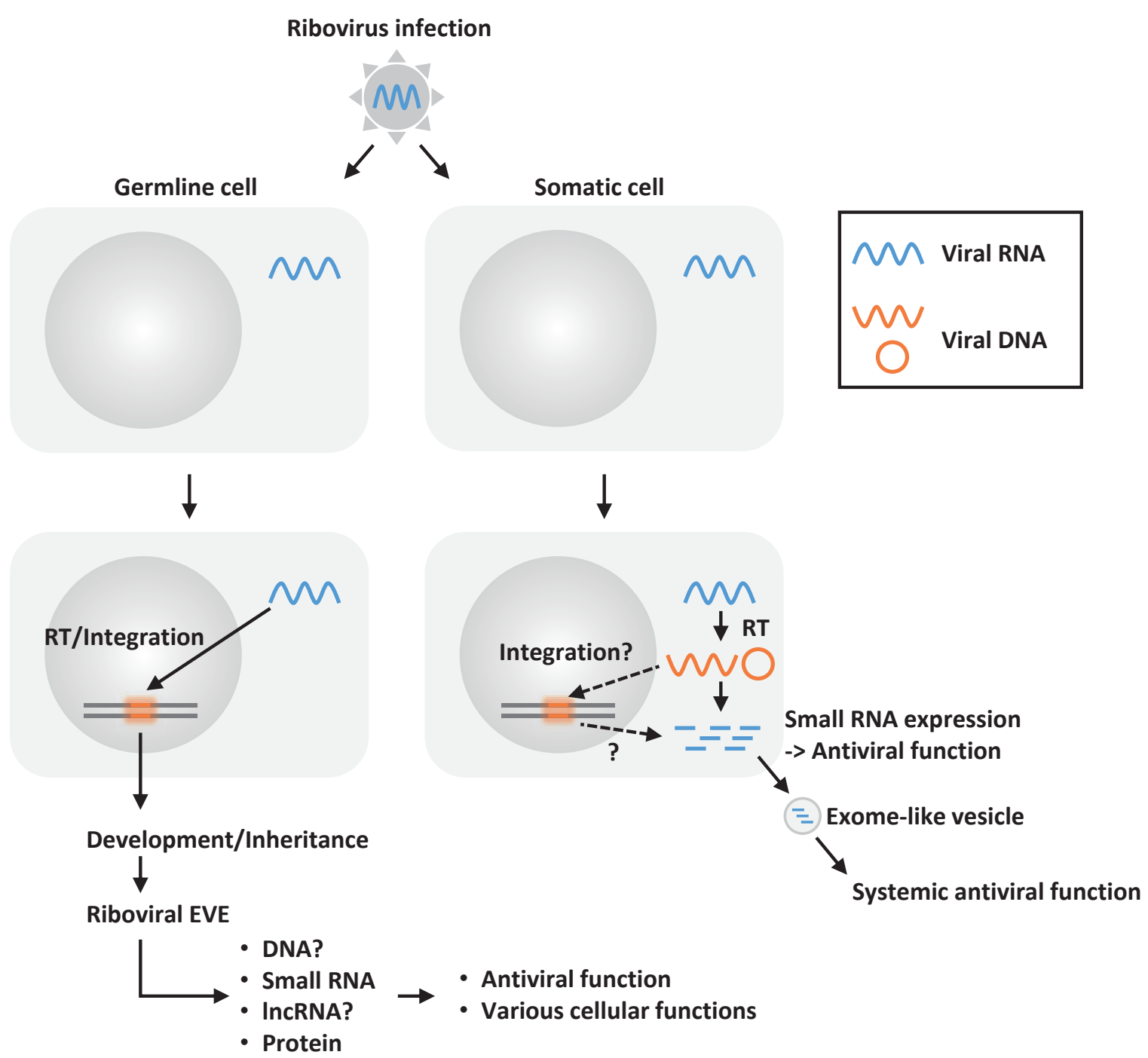

Fig. 4. Speculated and identified biological functions of reverse-transcribed riboviral sequences in their hosts. Current knowledge of speculated and identified biological functions of reverse-transcribed riboviral sequences and riboviral EVEs is shown. Question marks indicate speculative pathways or functions. Blue and orange lines show RNA and DNA molecules, respectively. RT, reverse transcription.

tion and increased mortality from viral infections. Importantly, AZT treatment did not affect viral titers, and thus Goic et al. concluded that vDNA biogenesis in mosquitoes is involved in tolerance to riboviral infections. The mechanism of vDNA formation and subsequent small RNA production seemed to be at least partially similar to that in Drosophila. Poirier et al. (2018) showed that cvDNA was produced in ribovirus-infected Aedes mosquitoes. Furthermore, high defective viral genome (DVG)containing virus stock showed lower infection rates in mosquitoes and treatment with $\mathrm{AZT}$ increased the infection rates of high DVG-containing viruses in mosquitoes. Thus, Poirier et al. proposed that DVGs can serve as templates for vDNA formation, which contributes to siRNA-mediated antiviral immune responses in insects.

Taken together, these data suggest that vDNA-mediated antiviral siRNA is an important antiviral pathway in fruit flies and mosquitoes. A series of findings regarding vDNA and small antiviral RNA formation opened the door for investigations into antiviral defenses in insects, which lack the RNA-dependent RNA polymerase that is involved in RNAi machineries in other eukaryotes such as nematodes and plants (Goic et al., 2013; Tassetto et al., 2017). Further studies in such insects should provide mechanistic insights into lineage-specific RNAi machineries.

\section{BIOLOGICAL FUNCTIONS OF GERMLINE- INTEGRATED RIBOVIRAL SEQUENCES}

In the genomes of many animals exist germlineintegrated riboviral sequences, called EVEs. Riboviral 
EVEs may function at the DNA, RNA or protein level (Fig. 4). As DNA elements, EVEs may act as regulators such as enhancers, promoters and insulators. EVEs may also express long non-coding RNAs or short RNAs, such as microRNAs, siRNA and piwi-interacting RNA (piRNA). Furthermore, EVEs may encode proteins with various functions. Below, I introduce several reported functions or possible functions of riboviral EVEs.

EVEs can encode proteins Genetic mutations and/or transposable element insertions can disrupt open reading frames (ORFs) within EVEs. Indeed, a large proportion of riboviral EVEs are thought to have been endogenized as far back as more than tens of MYA, and thus many have been pseudogenized. However, some EVEs still retain intact or relatively large ORFs. Although the presence of ORFs does not always mean that they encode proteins, such observations suggest that it is likely that these EVEs encode proteins in their hosts. Indeed, several riboviral EVEs have been shown to express proteins (Fujino et al., 2014; Kobayashi et al., 2016; Myers et al., 2016; Horie, 2017). Below, I summarize known and possible protein-encoding riboviral EVEs in animals.

Bornavirus-derived elements encoding proteins. Here, I will briefly consider the known and possible functions of bornavirus-derived EVEs (endogenous bornavirus-like elements, EBLs), which have been well described in several review papers (Honda and Tomonaga, 2016; Horie, 2017). Some EBLs were shown to express protein products in mammals, such as humans, ground squirrels and elephants (Ewing et al., 2007; Fujino et al., 2014; Kobayashi et al., 2016; Myers et al., 2016). Some of these seem to be functional in the cells: an EBL in humans is apparently involved in the regulation of genome stability (Myers et al., 2016), and another in thirteen-lined ground squirrels may function as an anti-bornaviral gene (Fujino et al., 2014). Several EBLs in bats of the genus Eptesicus and afrotherian animals (e.g., elephant) have also been strongly suggested to encode functional proteins, although their functions remain unclear (Horie et al., 2016; Kobayashi et al., 2016). These possibly functional EBLs are found in the genomes of non-model organisms, which makes it difficult to systematically study them. However, recent progress in sequencing technologies has made it easier to study such organisms than previously thought. Further work is needed to understand the functions of bornavirus-derived genes in vertebrates.

Filovirus-derived elements. Filoviruses belong to the family Filoviridae (Amarasinghe et al., 2018). Representative viruses in this family include Ebola virus (EBOV) and Marburg virus, which are highly pathogenic in humans and non-human primates (Amarasinghe et al.,
2018). EVEs derived from filoviruses are present in bats of the genus Myotis (Belyi et al., 2010b; Katzourakis and Gifford, 2010; Taylor et al., 2010, 2011). Among them, an element homologous to the VP35 gene of filoviruses, named endogenous filovirus-like VP35 (EFL35), potentially has interferon-antagonist activity. EFL35 was probably integrated into the bat genome more than 20 MYA and retains a relatively long ORF, which has evolved and been maintained under natural selection (Belyi et al., 2010b; Katzourakis and Gifford, 2010; Taylor et al., 2010, 2011). Kondoh et al. (2017) and Edwards et al. (2018) showed that EFL35 in Myotis bats (mEFL35) suppressed innate immune responses, as does the original protein VP35 of exogenous filoviruses. Kondoh et al. (2017) showed that exogenous expression of mEFL35 from $M$. lucifugus suppressed the antiviral signaling pathway in human cells. Edwards et al. (2018) further expanded this finding. They used mEFL35 proteins from 16 Myotis species, and revealed that many of them showed similar activities in human and Myotis bat cells. The biological significance of this interferon-antagonist activity is unclear, although one possible explanation is that mEFL35 is involved in modulating IFN and inflammatory responses (Edwards et al., 2018). However, the interferon-antagonist activity is not conserved in all mEFL35s: mEFL35 from several Myotis species lacked the activity (Edwards et al., 2018). Furthermore, transcripts from the mEFL35 locus have not yet even been detected. Further studies are needed to assess the biological impact of the interferon-antagonist activity of mEFL35 in bats.

In addition to the above effect on immune signaling, intriguingly, mEFL35 expression slightly decreased the polymerase activity of EBOV in a mini-replicon assay in human cells (Kondoh et al., 2017), although this effect was not observed in another study (Edwards et al., 2018). Because mEFL35 forms a hetero-oligomer with the VP35 protein of modern filoviruses, this interaction might contribute to the inhibition of polymerase activity: namely, mEFL35 might function as a dominant-negative mutant against the viral VP35 protein. Note that mEFL35 is distantly related to modern exogenous filoviruses including EBOV (the amino acid identity between EBOV VP35 and EFL35 is 32\%). Thus, this EFL35 might act as an antiviral gene against yet-to-be-discovered filoviruses that are genetically closer. Recently, two novel lineages of filoviruses, named Wenling frogfish filovirus and Wenling Thamnaconus septentrionalis filovirus, and Měnglà virus were detected in fishes and are distantly related to the three previously identified lineages of filoviruses (the genera Cuevavirus, Ebolavirus and Marburgvirus) (Shi et al., 2018). Although these two novel filoviruses are not genetically close to mEFL35, their discovery implies that additional filovirus lineages occur in nature. Thus, extensive surveillance of exogenous viruses will be impor- 
tant for understanding the function of riboviral EVEs, as previously discussed (Horie, 2017).

EVEs may function as small non-coding RNAs Several EVEs encode piRNA, a type of small non-coding RNA that is mainly involved in epigenetic and post-transcriptional suppression of retrotransposable elements (Siomi et al., 2011). piRNA precursors are usually expressed from clusters in genomes, called piRNA clusters (Lau et al., 2006; Aravin et al., 2007). Interestingly, some riboviral EVEs are present in such piRNA clusters, and piRNAs are expressed from these riboviral EVE loci in animals (Parrish et al., 2015; Suzuki et al., 2017; Whitfield et al., 2017).

piRNAs are expressed from bornavirus-derived EVE loci, at least in several primates and rodents (Parrish et al., 2015). In insects, piRNAs are expressed from EVEs derived from many riboviruses, such as flaviviruses, rhabdoviruses, bunyaviruses and reoviruses (Suzuki et al., 2017; Whitfield et al., 2017). These EVE-derived piRNAs were suggested to be involved in antiviral mechanisms. Parrish et al. (2015) showed that bornaviral EVEs accumulated in piRNA clusters independently in two different lineages of mammals (primates and rodents), prompting them to propose that there may have been survival advantages in animals with such EVEs in the piRNA cluster, probably attributable to antiviral piRNA expression. However, the antiviral activity of these EVE-derived piRNAs against modern viruses has not been shown thus far. Further virological studies are needed to understand the biological significance of piRNA-encoding riboviral EVEs.

\section{CONCLUSION AND FUTURE DIRECTIONS}

As described above, recent studies on the interactions among eukaryotes, retrotransposons and riboviruses have contributed to a deeper understanding of viral biology. However, many open questions remain. Although many riboviral EVEs have been detected in diverse eukaryotes, their integration mechanisms are not well studied, except for several examples described here. Thus, more detailed investigations, such as sequence-based and molecular biological analyses, are needed to clarify the integration mechanisms. Also, several hypothetical mechanisms of action of EVE-derived genes, such as those underlying the antiviral effect of EVE-derived piRNAs, have not yet been tested (Parrish et al., 2015; Miesen et al., 2016; Parrish and Tomonaga, 2016; Suzuki et al., 2017; Whitfield et al., 2017). One can also imagine that some viruses may counteract such antiviral mechanisms, as many mammalian viruses encode innate immunity-antagonist proteins. Thus, studies from such a viewpoint will be important for understanding the arms race between riboviruses and their hosts. Considering the diversity of eukaryotes, the findings presented here are just the tip of iceberg: many other interesting interactions probably occur among eukaryotes, retrotransposons and riboviruses in other eukaryotes, or even in the animals introduced here. Further studies will contribute to a deeper understanding of these tripartite interactions.

\section{CONFLICTS OF INTEREST}

None.

I thank ENDEAVR (the Endogenous Elements from Ancient Viruses Research Club) in Japan, which is partially supported by Cross-sectoral Research Platform Development Program 2016 of Kyoto University, for providing the opportunity to publish this review article. This work was supported by the Hakubi Project of Kyoto University; a Grant-in-Aid for Scientific Research on Innovative Areas from the Ministry of Education, Culture, Sports, Science and Technology (MEXT) of Japan (grant number 17H05821); and a JSPS KAKENHI for Challenging Research (Exploratory) (grant number 18K19443).

\section{REFERENCES}

Aiewsakun, P., and Katzourakis, A. (2015) Endogenous viruses: connecting recent and ancient viral evolution. Virology 479-480, 26-37.

Amarasinghe, G. K., Aréchiga Ceballos, N. G., Banyard, A. C., Basler, C. F., Bavari, S., Bennett, A. J., Blasdell, K. R., Briese, T., Bukreyev, A., Cai, Y., et al. (2018) Taxonomy of the order Mononegavirales: update 2018. Arch. Virol. 163, $2283-2294$.

Aravin, A. A., Sachidanandam, R., Girard, A., Fejes-Toth, K., and Hannon, G. J. (2007) Developmentally regulated piRNA clusters implicate MILI in transposon control. Science 316, 744-747.

Ballinger, M. J., Bruenn, J. A., and Taylor, D. J. (2012) Phylogeny, integration and expression of sigma virus-like genes in Drosophila. Mol. Phylogenet. Evol. 65, 251-258.

Beauregard, A., Curcio, M. J., and Belfort, M. (2008) The take and give between retrotransposable elements and their hosts. Annu. Rev. Genet. 42, 587-617.

Beck, C. R., Garcia-Perez, J. L., Badge, R. M., and Moran, J. V. (2011) LINE-1 elements in structural variation and disease. Annu. Rev. Genomics Hum. Genet. 12, 187-215.

Belyi, V. A., Levine, A. J., and Skalka, A. M. (2010a) Sequences from ancestral single-stranded DNA viruses in vertebrate genomes: the Parvoviridae and Circoviridae are more than 40 to 50 million years old. J. Virol. 84, 12458-12462.

Belyi, V. A., Levine, A. J., and Skalka, A. M. (2010b) Unexpected inheritance: multiple integrations of ancient bornavirus and ebolavirus/marburgvirus sequences in vertebrate genomes. PLoS Pathog. 6, e1001030.

Brouha, B., Schustak, J., Badge, R. M., Lutz-Prigge, S., Farley, A. H., Moran, J. V., and Kazazian, H. H., Jr. (2003) Hot L1s account for the bulk of retrotransposition in the human population. Proc. Natl. Acad. Sci. USA 100, 5280-5285.

Buzdin, A., Gogvadze, E., Kovalskaya, E., Volchkov, P., Ustyugova, S., Illarionova, A., Fushan, A., Vinogradova, T., and Sverdlov, E. (2003) The human genome contains many types of chimeric retrogenes generated through in vivo RNA recombination. Nucleic Acids Res. 31, 4385-4390.

Buzdin, A., Ustyugova, S., Gogvadze, E., Vinogradova, T., Lebedev, 
Y., and Sverdlov, E. (2002) A new family of chimeric retrotranscripts formed by a full copy of U6 small nuclear RNA fused to the 3' terminus of 11 . Genomics 80, 402-406.

Buzdin, A. A. (2004) Retroelements and formation of chimeric retrogenes. Cell. Mol. Life Sci. 61, 2046-2059.

Claverie, J. M., and Abergel, C. (2009) Mimivirus and its virophage. Annu. Rev. Genet. 43, 49-66.

Cordaux, R., and Batzer, M. A. (2009) The impact of retrotransposons on human genome evolution. Nat. Rev. Genet. 10, 691-703.

Cotton, J. A., Steinbiss, S., Yokoi, T., Tsai, I. J., and Kikuchi, T. (2016) An expressed, endogenous Nodavirus-like element captured by a retrotransposon in the genome of the plant parasitic nematode Bursaphelenchus xylophilus. Sci. Rep. 6, 39749 .

Coufal, N. G., Garcia-Perez, J. L., Peng, G. E., Yeo, G. W., Mu, Y., Lovci, M. T., Morell, M., O’Shea, K. S., Moran, J. V., and Gage, F. H. (2009) L1 retrotransposition in human neural progenitor cells. Nature 460, 1127-1131.

Crochu, S., Cook, S., Attoui, H., Charrel, R. N., De Chesse, R., Belhouchet, M., Lemasson, J. J., de Micco, P., and de Lamballerie, X. (2004) Sequences of flavivirus-related RNA viruses persist in DNA form integrated in the genome of Aedes spp. mosquitoes. J. Gen. Virol. 85, 1971-1980.

Daugherty, M. D., and Malik, H. S. (2012) Rules of engagement: molecular insights from host-virus arms races. Annu. Rev. Genet. 46, 677-700.

Denli, A. M., Narvaiza, I., Kerman, B. E., Pena, M., Benner, C., Marchetto, M. C., Diedrich, J. K., Aslanian, A., Ma, J., Moresco, J. J., et al. (2015) Primate-specific ORF0 contributes to retrotransposon-mediated diversity. Cell $\mathbf{1 6 3}$, 583-593.

Dewannieux, M., Dupressoir, A., Harper, F., Pierron, G., and Heidmann, T. (2004) Identification of autonomous IAP LTR retrotransposons mobile in mammalian cells. Nat. Genet. 36, 534-539.

Dupressoir, A., Marceau, G., Vernochet, C., Bénit, L., Kanellopoulos, C., Sapin, V., and Heidmann, T. (2005) Syncytin-A and syncytin-B, two fusogenic placenta-specific murine envelope genes of retroviral origin conserved in Muridae. Proc. Natl. Acad. Sci. USA 102, 725-730.

Edwards, M. R., Liu, H., Shabman, R. S., Ginell, G. M., Luthra, P., Ramanan, P., Keefe, L. J., Köllner, B., Amarasinghe, G. K., Taylor, D. J., et al. (2018) Conservation of structure and immune antagonist functions of filoviral VP35 homologs present in microbat genomes. Cell Rep. 24, 861-872. e6.

Eickbush, T. H., and Jamburuthugoda, V. K. (2008) The diversity of retrotransposons and the properties of their reverse transcriptases. Virus Res. 134, 221-234.

Esnault, C., Maestre, J., and Heidmann, T. (2000) Human LINE retrotransposons generate processed pseudogenes. Nat. Genet. 24, 363-367.

Ewing, R. M., Chu, P., Elisma, F., Li, H., Taylor, P., Climie, S., McBroom-Cerajewski, L., Robinson, M. D., O'Connor, L., Li, M., et al. (2007) Large-scale mapping of human proteinprotein interactions by mass spectrometry. Mol. Syst. Biol. 3, 89.

Feng, Q., Moran, J. V., Kazazian, H. H., Jr., and Boeke, J. D. (1996) Human L1 retrotransposon encodes a conserved endonuclease required for retrotransposition. Cell 87, 905-916.

Flint, S. J., Racaniello, V. R., Rall, G. F., Skalka, A. M., and Enquist, L. W. (2015) Principles of Virology, 4th ed. ASM Press, Washington, D. C., USA.

Fujino, K., Horie, M., Honda, T., Merriman, D. K., and Tomonaga, K. (2014) Inhibition of Borna disease virus replication by an endogenous bornavirus-like element in the ground squirrel genome. Proc. Natl. Acad. Sci. USA 111, 13175-13180.

Gammon, D. B., and Mello, C. C. (2015) RNA interferencemediated antiviral defense in insects. Curr. Opin. Insect Sci. 8, 111-120.

Garcia-Perez, J. L., Morell, M., Scheys, J. O., Kulpa, D. A., Morell, S., Carter, C. C., Hammer, G. D., Collins, K. L., O'Shea, K. S., Menendez, P., et al. (2010) Epigenetic silencing of engineered L1 retrotransposition events in human embryonic carcinoma cells. Nature 466, 769-773.

Geuking, M. B., Weber, J., Dewannieux, M., Gorelik, E., Heidmann, T., Hengartner, H., Zinkernagel, R. M., and Hangartner, L. (2009) Recombination of retrotransposon and exogenous RNA virus results in nonretroviral cDNA integration. Science 323, 393-396.

Gilbert, N., Lutz-Prigge, S., and Moran, J. V. (2002) Genomic deletions created upon LINE-1 retrotransposition. Cell 110, 315-325.

Goic, B., Stapleford, K. A., Frangeul, L., Doucet, A. J., Gausson, V., Blanc, H., Schemmel-Jofre, N., Cristofari, G., Lambrechts, L., Vignuzzi, M., et al. (2016) Virus-derived DNA drives mosquito vector tolerance to arboviral infection. Nat. Commun. 7, 12410.

Goic, B., Vodovar, N., Mondotte, J. A., Monot, C., Frangeul, L., Blanc, H., Gausson, V., Vera-Otarola, J., Cristofari, G., and Saleh, M. C. (2013) RNA-mediated interference and reverse transcription control the persistence of RNA viruses in the insect model Drosophila. Nat. Immunol. 14, 396-403.

Hancks, D. C., and Kazazian, H. H., Jr. (2012) Active human retrotransposons: variation and disease. Curr. Opin. Genet. Dev. 22, 191-203.

Hancks, D. C., and Kazazian, H. H., Jr. (2016) Roles for retrotransposon insertions in human disease. Mob. DNA 7, 9.

Havecker, E. R., Gao, X., and Voytas, D. F. (2004) The diversity of LTR retrotransposons. Genome Biol. 5, 225.

Hoffmann, H. H., Schneider, W. M., and Rice, C. M. (2015) Interferons and viruses: an evolutionary arms race of molecular interactions. Trends Immunol. 36, 124-138.

Honda, T., and Tomonaga, K. (2016) Endogenous non-retroviral RNA virus elements evidence a novel type of antiviral immunity. Mob. Genet. Elements 6, e1165785.

Horie, M. (2017) The biological significance of bornavirus-derived genes in mammals. Curr. Opin. Virol. 25, 1-6.

Horie, M., Honda, T., Suzuki, Y., Kobayashi, Y., Daito, T., Oshida, T., Ikuta, K., Jern, P., Gojobori, T., Coffin, J. M., et al. (2010) Endogenous non-retroviral RNA virus elements in mammalian genomes. Nature 463, 84-87.

Horie, M., Kobayashi, Y., Honda, T., Fujino, K., Akasaka, T., Kohl, C., Wibbelt, G., Mühldorfer, K., Kurth, A., Müller, M. A., et al. (2016) An RNA-dependent RNA polymerase gene in bat genomes derived from an ancient negative-strand RNA virus. Sci. Rep. 6, 25873.

Horie, M., Kobayashi, Y., Suzuki, Y., and Tomonaga, K. (2013) Comprehensive analysis of endogenous bornavirus-like elements in eukaryote genomes. Philos. Trans. R. Soc. Lond. B Biol. Sci. 368, 20120499.

Horie, M., and Tomonaga, K. (2011) Non-retroviral fossils in vertebrate genomes. Viruses 3, 1836-1848.

Horie, M., and Tomonaga, K. (2019) Paleovirology of bornaviruses: what can be learned from molecular fossils of bornaviruses. Virus Res. 262, 2-9.

International Human Genome Sequencing Consortium (IHGSC) (2001) Initial sequencing and analysis of the human genome. Nature 409, 860-921.

Katzourakis, A., and Gifford, R. J. (2010) Endogenous viral ele- 
ments in animal genomes. PLoS Genet. 6, e1001191.

Klenerman, P., Hengartner, H., and Zinkernagel, R. M. (1997) A non-retroviral RNA virus persists in DNA form. Nature 390, 298-301.

Kobayashi, Y., Horie, M., Nakano, A., Murata, K., Itou, T., and Suzuki, Y. (2016) Exaptation of bornavirus-like nucleoprotein elements in afrotherians. PLoS Pathog. 12, e1005785.

Kondoh, T., Manzoor, R., Nao, N., Maruyama, J., Furuyama, W., Miyamoto, H., Shigeno, A., Kuroda, M., Matsuno, K., Fujikura, D., et al. (2017) Putative endogenous filovirus VP35-like protein potentially functions as an IFN antagonist but not a polymerase cofactor. PLoS One 12, e0186450.

Koyama, A. H. (1995) Induction of apoptotic DNA fragmentation by the infection of vesicular stomatitis virus. Virus Res. 37, 285-290.

Kuff, E. L., and Lueders, K. K. (1988) The intracisternal Aparticle gene family: structure and functional aspects. Adv. Cancer Res. 51, 183-276.

La Scola, B., Desnues, C., Pagnier, I., Robert, C., Barrassi, L., Fournous, G., Merchat, M., Suzan-Monti, M., Forterre, P., Koonin, E., et al. (2008) The virophage as a unique parasite of the giant mimivirus. Nature 455, 100-104.

Lau, N. C., Seto, A. G., Kim, J., Kuramochi-Miyagawa, S., Nakano, T., Bartel, D. P., and Kingston, R. E. (2006) Characterization of the piRNA complex from rat testes. Science 313, 363-367.

Malik, H. S., Henikoff, S., and Eickbush, T. H. (2000) Poised for contagion: evolutionary origins of the infectious abilities of invertebrate retroviruses. Genome Res. 10, 1307-1318.

Maori, E., Tanne, E., and Sela, I. (2007) Reciprocal sequence exchange between non-retro viruses and hosts leading to the appearance of new host phenotypes. Virology 362, 342-349.

Martin, S. L. (2006) The ORF1 protein encoded by LINE-1: structure and function during L1 retrotransposition. J. Biomed. Biotechnol. 2006, 45621.

Mi, S., Lee, X., Li, X., Veldman, G. M., Finnerty, H., Racie, L., LaVallie, E., Tang, X. Y., Edouard, P., Howes, S., et al. (2000) Syncytin is a captive retroviral envelope protein involved in human placental morphogenesis. Nature 403, 785-789.

Miesen, P., Joosten, J., and van Rij, R. P. (2016) PIWIs go viral: arbovirus-derived piRNAs in vector mosquitoes. PLoS Pathog. 12, e1006017.

Mills, R. E., Bennett, E. A., Iskow, R. C., and Devine, S. E. (2007) Which transposable elements are active in the human genome? Trends Genet. 23, 183-191.

Morrish, T. A., Gilbert, N., Myers, J. S., Vincent, B. J., Stamato, T. D., Taccioli, G. E., Batzer, M. A., and Moran, J. V. (2002) DNA repair mediated by endonuclease-independent LINE-1 retrotransposition. Nat. Genet. 31, 159-165.

Muotri, A. R., Chu, V. T., Marchetto, M. C. N., Deng, W., Moran, J. V., and Gage, F. H. (2005) Somatic mosaicism in neuronal precursor cells mediated by L1 retrotransposition. Nature 435, 903-910.

Myers, K. N., Barone, G., Ganesh, A., Staples, C. J., Howard, A. E., Beveridge, R. D., Maslen, S., Skehel, J. M., and Collis, S. J. (2016) The bornavirus-derived human protein EBLN1 promotes efficient cell cycle transit, microtubule organisation and genome stability. Sci. Rep. 6, 35548 .

Ono, R., Nakamura, K., Inoue, K., Naruse, M., Usami, T., Wakisaka-Saito, N., Hino, T., Suzuki-Migishima, R., Ogonuki, N., Miki, H., et al. (2006) Deletion of Peg10, an imprinted gene acquired from a retrotransposon, causes early embryonic lethality. Nat. Genet. 38, 101-106.

Parrish, N. F., Fujino, K., Shiromoto, Y., Iwasaki, Y. W., Ha, H.,
Xing, J., Makino, A., Kuramochi-Miyagawa, S., Nakano, T., Siomi, H., et al. (2015) piRNAs derived from ancient viral processed pseudogenes as transgenerational sequence-specific immune memory in mammals. RNA 21, 1691-1703.

Parrish, N. F., and Tomonaga, K. (2016) Endogenized viral sequences in mammals. Curr. Opin. Microbiol. 31, 176183.

Pearce, A. F., and Lyles, D. S. (2009) Vesicular stomatitis virus induces apoptosis primarily through Bak rather than Bax by inactivating Mcl-1 and Bcl-X $\mathrm{L}_{\mathrm{L}}$. J. Virol. 83, 9102-9112.

Pizarro, J. G., and Cristofari, G. (2016) Post-Transcriptional control of LINE-1 retrotransposition by cellular host factors in somatic cells. Front. Cell Dev. Biol. 4, 14.

Platt, R. N., 2nd, and Ray, D. A. (2012) A non-LTR retroelement extinction in Spermophilus tridecemlineatus. Gene 500, 47-53.

Poirier, E. Z., Goic, B., Tomé-Poderti, L., Frangeul, L., Boussier, J., Gausson, V., Blanc, H., Vallet, T., Loyd, H., Levi, L. I., et al. (2018) Dicer-2-dependent generation of viral DNA from defective genomes of RNA viruses modulates antiviral immunity in insects. Cell Host Microbe 23, 353-365. e8.

Saleh, M. C., Tassetto, M., van Rij, R. P., Goic, B., Gausson, V., Berry, B., Jacquier, C., Antoniewski, C., and Andino, R. (2009) Antiviral immunity in Drosophila requires systemic RNA interference spread. Nature 458, 346-350.

Saleh, M. C., van Rij, R. P., Hekele, A., Gillis, A., Foley, E., O'Farrell, P. H., and Andino, R. (2006) The endocytic pathway mediates cell entry of dsRNA to induce RNAi silencing. Nat. Cell Biol. 8, 793-802.

Sassaman, D. M., Dombroski, B. A., Moran, J. V., Kimberland, M. L., Naas, T. P., DeBerardinis, R. J., Gabriel, A., Swergold, G. D., and Kazazian, H. H., Jr. (1997) Many human L1 elements are capable of retrotransposition. Nat. Genet. 16, $37-43$.

Sekita, Y., Wagatsuma, H., Nakamura, K., Ono, R., Kagami, M., Wakisaka, N., Hino, T., Suzuki-Migishima, R., Kohda, T., Ogura, A., et al. (2008) Role of retrotransposon-derived imprinted gene, Rtl1, in the feto-maternal interface of mouse placenta. Nat. Genet. 40, 243-248.

Shi, M., Lin, X. D., Chen, X., Tian, J. H., Chen, L. J., Li, K., Wang, W., Eden, J. S., Shen, J. J., Liu, L., et al. (2018) The evolutionary history of vertebrate RNA viruses. Nature 556, 197-202.

Shimizu, A., Nakatani, Y., Nakamura, T., Jinno-Oue, A., Ishikawa, O., Boeke, J. D., Takeuchi, Y., and Hoshino, H. (2014) Characterisation of cytoplasmic DNA complementary to non-retroviral RNA viruses in human cells. Sci. Rep. 4, 5074.

Siomi, M. C., Sato, K., Pezic, D., and Aravin, A. A. (2011) PIWI-interacting small RNAs: the vanguard of genome defence. Nat. Rev. Mol. Cell Biol. 12, 246-258.

Smith, R. H., Hallwirth, C. V., Westerman, M., Hetherington, N. A., Tseng, Y. S., Cecchini, S., Virag, T., Ziegler, M. L., Rogozin, I. B., Koonin, E. V., et al. (2016) Germline viral "fossils" guide in silico reconstruction of a mid-Cenozoic era marsupial adeno-associated virus. Sci. Rep. 6, 28965.

Suzuki, Y., Frangeul, L., Dickson, L. B., Blanc, H., Verdier, Y., Vinh, J., Lambrechts, L., and Saleh, M. C. (2017) Uncovering the repertoire of endogenous flaviviral elements in Aedes mosquito genomes. J. Virol. 91, e00571-17.

Suzuki, Y., Kobayashi, Y., Horie, M., and Tomonaga, K. (2014) Origin of an endogenous bornavirus-like nucleoprotein element in thirteen-lined ground squirrels. Genes Genet. Syst. 89, 143-148.

Tassetto, M., Kunitomi, M., and Andino, R. (2017) Circulating 
immune cells mediate a systemic RNAi-based adaptive antiviral response in Drosophila. Cell 169, 314-325. e13.

Taylor, D. J., Dittmar, K., Ballinger, M. J., and Bruenn, J. A. (2011) Evolutionary maintenance of filovirus-like genes in bat genomes. BMC Evol. Biol. 11, 336.

Taylor, D. J., Leach, R. W., and Bruenn, J. (2010) Filoviruses are ancient and integrated into mammalian genomes. BMC Evol. Biol. 10, 193.

tenOever, B. R. (2016) The evolution of antiviral defense systems. Cell Host Microbe 19, 142-149.

Tomonaga, K., Kobayashi, T., and Ikuta, K. (2002) Molecular and cellular biology of Borna disease virus infection. Microbes Infect. 4, 491-500.
Whitfield, Z. J., Dolan, P. T., Kunitomi, M., Tassetto, M., Seetin, M. G., Oh, S., Heiner, C., Paxinos, E., and Andino, R. (2017) The diversity, structure, and function of heritable adaptive immunity sequences in the Aedes aegypti Genome. Curr. Biol. 27, 3511-3519. e7.

Wilson, R. C., and Doudna, J. A. (2013) Molecular mechanisms of RNA interference. Annu. Rev. Biophys. 42, 217-239.

Yang, N., and Kazazian, H. H., Jr. (2006) L1 retrotransposition is suppressed by endogenously encoded small interfering RNAs in human cultured cells. Nat. Struct. Mol. Biol. 13, 763-771.

Zhdanov, V. M. (1975) Integration of viral genomes. Nature 256, 471-473. 